\title{
Current understanding of cancer-intrinsic PD-L1: regulation of expression and its protumoral activity
}

\author{
Pedram Yadollahi ${ }^{1,2, \#}$, You-Kyoung Jeon ${ }^{1,2, \#}$, Wooi Loon $\mathrm{Ng}^{1, \#} \mathcal{E}$ Inhak Choi ${ }^{1,2, *}$ \\ ${ }^{1}$ Innovative Therapeutic Research Institute, Inje University, Busan 47397, ${ }^{2}$ Department of Microbiology and Immunology, Inje University \\ College of Medicine, Busan 47392, Korea
}

In the last decade, we have witnessed an unprecedented clinical success in cancer immunotherapies targeting the programmed cell-death ligand 1 (PD-L1) and programmed cell-death 1 (PD-1) pathway. Besides the fact that PD-L1 plays a key role in immune regulation in tumor microenvironment, recently a plethora of reports has suggested a new perspective of non-immunological functions of PD-L1 in the regulation of cancer intrinsic activities including mesenchymal transition, glucose and lipid metabolism, stemness, and autophagy. Here we review the current understanding on the regulation of expression and intrinsic protumoral activity of cancer-intrinsic PD-L1. [BMB Reports 2021; 54(1): 12-20]

\section{INTRODUCTION}

Immune checkpoints are key players in immune regulation whose primary function is to maintain the immune homeostasis of the host, keeping the host protected from unwanted immune attacks in normal physiologic condition (1). Immune checkpoints regulate the magnitude and breadth of immune response and its dysregulation often causes an uncontrolled inflammatory reaction that leads to severe tissue damage. Checkpoint ligands and receptors come into play to sculpt immune responses by fine tuning the regulatory process involved in the development, activation, and differentiation of immune cells.

Among the co-inhibitory checkpoint ligands, programmed cell-death 1 ligand (PD-L1, B7-H1, CD274), is most comprehensively studied in terms of the molecular mechanisms behind its expression, immune regulation, and the clinical applications. PD-L1 suppresses T-cell immunity by means of interaction with

*Corresponding author. Tel: +82-51-890-5935; Fax: +82-51-890-5938; E-mail: miccih@inje.ac.kr

${ }^{\#}$ These authors contributed equally to this work.

https://doi.org/10.5483/BMBRep.2021.54.1.241

Received 20 October 2020, Revised 23 November 2020, Accepted 30 November 2020

Keywords: Cancer-intrinsic PD-L1, Protumoral activity, Regulation of expression, Signaling pathway, Signalosome receptors on T cells, such as programmed cell death 1 (PD-1) and CD80 (B7.1), leading to the inhibition of T-cell cytokine production and the induction of anergy, Treg, and apoptosis (2).

FDA-approved PD-1/PD-L1 inhibitors have shown durable and effective clinical responses in multiple advanced carcinomas and hematological malignancies (3). In the past decade, however, the initial excitement due to unprecedented clinical outcomes has been undermined by the unsatisfactory clinical observation that only a subset of patients respond to inhibitors across the cancer types, and most patients do not respond to or are resistant to the therapy (4).

A growing body of evidence indicates that the unresponsiveness and refractoriness to PD-1/PD-L1 blockade therapy is traced to the role of cancer-intrinsic PD-L1. Chen and his colleagues first suggested that PD-L1 expressed on cancer cells could act as a counter-receptor that can transmit an anti-apoptotic signal into the cancer cell, leading to the resistance to the T-cell attack (5). In line with this perspective, growing evidence has revealed that cancer-intrinsic PD-L1 effects the cancer progression in immune-deficient syngeneic or xenograft mouse models (6). The result indicates that cancer-intrinsic PD-L1 exerts protumoral activity, providing a new angle on a comprehensive biology of cancer-intrinsic PD-L1 as well as a new approach to overcome the resistance to the checkpoint-blockade therapy.

In this review, we cover the current biological principles that control PD-L1 expression in cancer cells and describe the protumoral activities of cancer-intrinsic PD-L1.

\section{REGULATION OF PD-L1 EXPRESSION}

Since we reported that IFN- $\gamma$ induces PD-L1 in cancer cells by means of the JAK-STAT-IRF-1 pathway (7), a plethora of studies has revealed several mechanisms behind the regulation of PD-L1 expression in a wide variety of cancer types. In this review, we summarize the current understanding of how the expression of PD-L1 is regulated in a variety of cancer cells.

\section{Genomic alteration}

Recently, gene structure analyses have shown that the genetic aberration at the PD-L1 gene locus is associated with upregulation of PD-L1 transcripts and proteins in various cancer types. 
The genetic alterations can be summarized as follows:

First, amplification of the PD-L1 gene at p24.1 on chromosome 9 leads to PD-L1 upregulation in breast cancer, lymphoma, lung cancer, and cervical cancer (8). Second, the translocation of class II transactivator (CIITA), a master regulator of the major histocompatibility complex, to the PD-L1 gene, forming a novel fusion transcript of CIITA-PD-L1 on 9p24.1, leads to overexpression of PD-L1 in primary mediastinal large B-cell lymphoma (PMBCL) (9). Third, truncation of the $3^{\prime}$ untranslated region (UTR) of PD-L1 gives rise to a longer stability of PD-L1 $\mathrm{mRNA}$, consequently leading to greater expression of PD-L1 (10). In addition, single nucleotide polymorphisms (SNPs) with AA+AG genotype of rs2297136 in 3'-UTR of the PD-L1 are correlated with PD-L1 expression in gastric cancers (GC) (11). Fourth, PD-L1 expression can be epigenetically regulated. For examples, the enhancer of zeste 2 polycomb repressive complex 2 subunit (EZH2), an epigenetic modifier, negatively regulates PD-L1 expression by upregulating H3K27me3 levels of PD-L1 promoter and interferon-responsible factor 1 (IRF-1) in hepatocellular carcinoma (12). In contrast, demethylation of $\mathrm{CpG}$ islands in the PD-L1 promoter is positively correlated with PD-L1 upregulation in breast cancer (13). It is noteworthy that methylation of histone in the promoter region of PD-L1 does not always inhibit PD-L1 expression. A mixed lineage leukemia protein-1 (MLL1), a member of the evolutionarily conserved SET1 family of histone $\mathrm{H} 3$ lysine 4 (H3K4) methyltransferases, directly binds to PD-L1 promoter to increase PD-L1 transcription by catalyzing H3K4me3 formation (14).

\section{Aberrant oncogenic signaling pathways}

Aberrant oncogenic signaling pathways play an active role in proliferation, survival, and differentiation of cancer cells. Certain oncogenic pathways contribute to promoting cancer progression as well as driving PD-L1 expression. Some oncogenic transcription factors (TF) are believed to positively regulate PD-L1 transcription by directly binding to the PD-L1 promoter. Overexpressed MYC, HIF-1 $\alpha$ and HIF-2 $\alpha$ come into play for PD-L1 upregulation in melanoma, NSCLC, lymphoma, head and neck squamous cell carcinoma (HNSCC), and breast cancers, whereas activating mutation of NF-kB and STAT3 (p.E696K) can target PD-L1 promoter in the absence of cytokine to upregulate the PD-L1 expression in melanoma and NK/T cell lymphoma (NKTL) (15-18). In addition, constitutively activated AP-1 increases PD-L1 expression by binding to the tandem AP-1 binding sites on the PD-L1 promoter in classical Hodgkin's lymphoma (19).

The oncogenic MEK-ERK signaling pathway, playing a key role in cancer-cell survival and proliferation, leads to PD-L1 expression in a variety of cancer types (20). Activating mutations of KRAS, BRAF, and EGFR can drive PD-L1 expression by means of the MEK-ERK pathway in conjunction with activation of STAT1 (21). Particularly, the KRAS mutation-mediated MEK-ERK pathway can cause phosphorylation and subsequent inhibition of tristetraprolin (TTP) that targets AU-rich element in the 3'-UTR of PD-L1 mRNA, ultimately leading to robust stability of PD-L1 mRNA (22). Oncogenic fusion proteins, such as NPManaplastic lymphoma kinase (NPM-ALK) and EML4-ALK fusion protein, often produce constitutively active ALK and subsequently lead to PD-L1 expression by means of $\mathrm{p}$-ERK1/2 and $\mathrm{p}$-AKT (23). Recently, the Hippo pathway was described as regulating PD-L1 expression. When the Hippo pathway is off, unphosphorylated TAZ/YAP translocates into the nucleus, forming a complex with TEAD and increases PD-L1 transcription. However, when the Hippo pathway is on, phosphorylated upstream kinases, including MST1/MST2 and LATS1/LATS2, phosphorylate and subsequently degrade TAZ/YAP in the cytosol, leading to the shutdown of PD-L1 expression (24).

Some of the tumor suppressor genes are involved in PD-L1 expression by means of different signaling pathways. Loss of the tumor suppressor PTEN, a negative regulator of PI3K, strongly sustains PD-L1 upregulation via activation of the PI3K-AKTmTOR (mTORC1) axis (25). Wild-type and mutated p53 can positively regulate IFN- $\gamma$-induced PD-L1 expression in melanoma by boosting JAK2 expression, whereas p53 can downregulate PD-L1 via miR-34a in NSCLC, a discrepant observation on the effect of p53 on PD-L1 expression depending on the context $(26,27)$. The presence of tumor suppressor TET2 mediates PD-L1 expression via the IFN- $\gamma$-JAK2-STAT1 axis (28). In contrast, loss of tumor suppressor IRF-1 is associated with PD-L1 downregulation caused by loss of its antagonistic activity against IRF-2, a transcription repressor of PD-L1 $(29,30)$. Similarly, loss of the tumor suppressor cyclin-dependent kinase 5 (CDK5) diminishes PD-L1 expression via promoting expression of PD-L1 repressors, including IRF-2 and IRF2BP2 (31).

Regulation by post-transcriptional/translational modification Both post-transcriptional and post-translational modifications are essential to maintain the homeostasis of gene expressions and diversify their protein functions, which are modulated by microRNAs (miRNAs) that target 3'-UTR of the gene and modification processes, including glycosylation, phosphorylation, ubiquitination, sumoylation (SUMO), and acetylation (32). Accumulating reports have pointed out that multiple miRNAs directly or indirectly regulate the levels of PD-L1 transcripts across a variety of cancers, as summarized in Table 1.

Long non-coding RNAs (IncRNA) are a class of transcripts longer than 200 nucleotides, functioning as a competing endogenous RNAs (ceRNA) by competitively binding to miRNAs, thus negatively regulating miRNA expression. LncRNAs, such as metastasis-associated lung adenocarcinoma transcript 1 (MALAT1) and SNHG14, can sponge miRNAs that target PD-L1 mRNA $(33,34)$. A newly identified IncRNA IFN-stimulated non-coding RNA 1 (INCR1) enables PD-L1 induction by binding and inhibiting HNRNPH1, a nuclear ribonucleoprotein, that negatively regulates PD-L1 transcripts (35).

$\mathrm{N}$-glycosylated PD-L1 is more stable from proteasome-mediated degradation than is non-glycosylated PD-L1. Glycogen synthase kinase $3 \beta$ (GSK3 $\beta$ ) binds to and phosphorylates nonglycosylated PD-L1, further triggering interaction with E3 ligase 
Table 1. Regulation of cancer-intrinsic PD-L1 expression by miRNA, cytokine/chemokines, viral infection, chemotherapy, and radiation

\begin{tabular}{|c|c|c|c|}
\hline Category & Key molecules/signaling pathways involved & Cancer cell type & Reference \\
\hline $\begin{array}{l}\text { miRNAs directly targeting } \\
\text { PD-L1 mRNA }\end{array}$ & $\begin{array}{l}\text { miR-15a/16/193a, miR-17-5p, miR-18, miR-33a, miR-34a, } \\
\text { miR-93/106b/142-5p, miR-138-5p, miR-140/142/340/383, miR-146a, } \\
\text { miR-148a-3p, miR-152, miR-155-5p, miR-195, miR-197, miR-200/152, } \\
\text { miR-375, miR-424, miR-873, miR-497-5p, miR-3127-5p miR-3609 }\end{array}$ & $\begin{array}{l}\text { AML, BC, CC, CRC, GC, } \\
\text { HCC, LUAD, MPM, } \\
\text { NSCLC, PC, melanoma, } \\
\text { laryngeal cancer }\end{array}$ & $(77,78)$ \\
\hline $\begin{array}{l}\text { miRNAs targeting } \\
\text { signaling pathways that } \\
\text { regulate PD-L1 } \\
\text { expression }\end{array}$ & $\begin{array}{l}\text { 1) PTEN: miR-10a, miR-19, miR-21, miR-20b, miR-221 } \\
\text { 2) IRF: miR-23, miR-383 } \\
\text { 3) mTOR: miR-100, miR-101, miR-497, miR-1999a-3p } \\
\text { 4) STAT-1: miR-27, miR-145, miR-146a, miR-150 } \\
\text { 5) EIF4B: miR-150, miR-216a }\end{array}$ & $\begin{array}{l}\text { CC, CRC, GC, } \\
\text { NPC, NSCLC, OC }\end{array}$ & $(79,80)$ \\
\hline $\begin{array}{l}\text { Inflammatory cytokine } \\
\text { receptor signaling }\end{array}$ & $\begin{array}{l}\text { A. Inflammatory cytokines: } \\
\text { IFN- } \gamma \text {, IFN- } \alpha / \beta \text {, TNF- } \alpha \text {, TGF- } \beta \text {, IL-1 } \beta \text {, IL-4, IL-6, IL-10, IL-12, IL-17, IL-27, } \\
\text { CXCL9/10/11, TLR3, TLR4 } \\
\text { B. Inflammatory cytokine receptor signaling pathways: } \\
\text { 1) JAK2/STAT1/IRF-1, 2) JAK/STAT3, 3) RAS/BRAF/MEK/ERK, 4) NF-kB, } \\
\text { 5) TLR/MyD88/TRAF6, 6) PI3K/AKT/mTOR }\end{array}$ & $\begin{array}{l}\text { CRC, GC, OC, PC, RCC, } \\
\text { lung cancer, multiple } \\
\text { myeloma }\end{array}$ & $(78,81)$ \\
\hline $\begin{array}{l}\text { Insulin, epithelial growth } \\
\text { factor (EGF) }\end{array}$ & $\begin{array}{l}\text { 1) PI3K/AKT/mTOR pathway } \\
\text { 2) Increase PD-L1 transport or stability }\end{array}$ & CRC & (82) \\
\hline Epstein-Barr virus (EBV) & $\begin{array}{l}\text { 1) Repression of miR-34a via EBNA2 and EBF1 (upregulating PD-L1) } \\
\text { 2) miR-BHRF 1-2-5p (down-regulating PD-L1) } \\
\text { 3) Amplification of PD-L1 gene locus in 9p24.1 site } \\
\text { 4) EBNA1-mediated JAK2/STAT1/IFR-1/PD-L1 pathway }\end{array}$ & $\begin{array}{l}\text { GC, Burkitt lymphoma, } \\
\text { diffuse large } \\
\text { B cell lymphoma }\end{array}$ & $(83-86)$ \\
\hline $\begin{array}{l}\text { Human papilloma virus } \\
\text { (HPV) }\end{array}$ & $\begin{array}{l}\text { 1) E5-mediated EGFR-, EGFR/YAP-dependent } \\
\text { 2) E6-mediated p53-miR34a/200c-dependent } \\
\text { 3) E7-mediated Rb-NF-кB dependent }\end{array}$ & CC, lung cancer & (87) \\
\hline $\begin{array}{l}\text { Kaposi's } \\
\text { sarcoma-associated } \\
\text { herpesvirus (KSHV) }\end{array}$ & $\begin{array}{l}\text { 1) NF-kB-MAPK, AKT pathway } \\
\text { 2) Increasing IL-1 } \beta\end{array}$ & Lymphoma & (88) \\
\hline Cytotoxic drug & $\begin{array}{l}\text { 1) JAK2/STAT1 pathway (5-Fluorouracil, Gencitabine, Paclitaxel) } \\
\text { 2) HIF-1 } \alpha \text {-mediated (Carboplatin, Doxorubicin, Gencitabine, Paclitaxel) }\end{array}$ & $\begin{array}{l}\text { PC, triple-negative } \\
\text { breast cancer (TNBC) }\end{array}$ & $(89,90)$ \\
\hline Radiation & $\begin{array}{l}\text { 1) cGAS/STING cascade, ATMATR/CHK1 pathway } \\
\text { 2) IL-6-MEK/ERK pathway }\end{array}$ & $\begin{array}{l}\text { PC, lung cancer, } \\
\text { osteosarcoma }\end{array}$ & $(91,92)$ \\
\hline
\end{tabular}

AML, acute myelocytic leukemia; BC, breast cancer; CC, cervical cancer; CRC, colorectal carcinoma; GC, gastric cancer; HCC, hepatocellular carcinoma; LUAD, lung adenocarcinoma; MPM, malignant pleural mesothelioma; NSCLC, non-small cell lung carcinoma; OC, ovarian cancer; PC, pancreatic cancer; RCC, renal cell carcinoma.

to result in phosphorylation-dependent degradation by proteasome (36). However, the IL-6/JAK1 pathway increases PD-L1 stability by phosphorylating PD-L1 Tyr112, recruiting endoplasmic reticulum-associated $\mathrm{N}$-glycosyltransferase STT3A that leads to PD-L1 glycosylation and consequent PD-L1 protein stability (37).

In addition, E3 ubiquitin ligase STIP1 homology and U-box containing protein 1 (STUB1) act as a negative regulator of PD-L1 expression via mediating proteasomal degradation of the IFN$\gamma$ R1/JAK1 complex (38). Recently, CMTM6, a type-3 transmembrane protein, was described as a co-localizing partner protein for PD-L1 on the plasma membrane and in a recycling endosome, increasing the half-life of PD-L1 by preventing PD-L1 ubiquitination and subsequent degradation $(39,40)$. TNF- $\alpha$ within the tumor microenvironment stabilizes PD-L1 expression by means of COP9 signalosome 5 (CSN5) that directly deubiquitinates PD-L1 and impedes degradation of PD-L1 (41). Cyclin D-CDK4 and the cullin 3-SPOP E3 ligase is also responsible for the downregulation of PD-L1 expression by means of protea- some-mediated degradation of PD-L1 protein (42). Interestingly, IncRNAs can also control PD-L1 levels by stabilizing PD-L1 protein. MIR17HG directly binds to PD-L1 and stabilizes the PD-L1 protein in CRC (43). A novel IncRNA GATA3-AS1 can also stabilize PD-L1 protein by preventing PD-L1 ubiquitination by means of the miR-676-3p/COPS5 axis in TNBC (44).

Accumulating evidence indicates that PD-L1 expression can be influenced by a variety of entities, such as cytotoxic chemotherapy, radiation, cytokines/chemokines from tumor microenvironment (TME), and virus infection. The mechanisms and cancer types whereby PD-L1 expression is regulated by these entities are summarized in Table 1.

Collectively, expression of cancer-intrinsic PD-L1 can be regulated by multiple mechanisms, including genetic alteration of PD-L1 gene locus, aberrant oncogenic signaling pathways, posttranscriptional and posttranslational modification, TME cytokines/ chemokines, cytotoxic therapies, and oncogenic viruses (Fig. 1). Thus, the relevant pathways may be targeted with small molecules to control PD-L1 expression in cancer cells, which might 


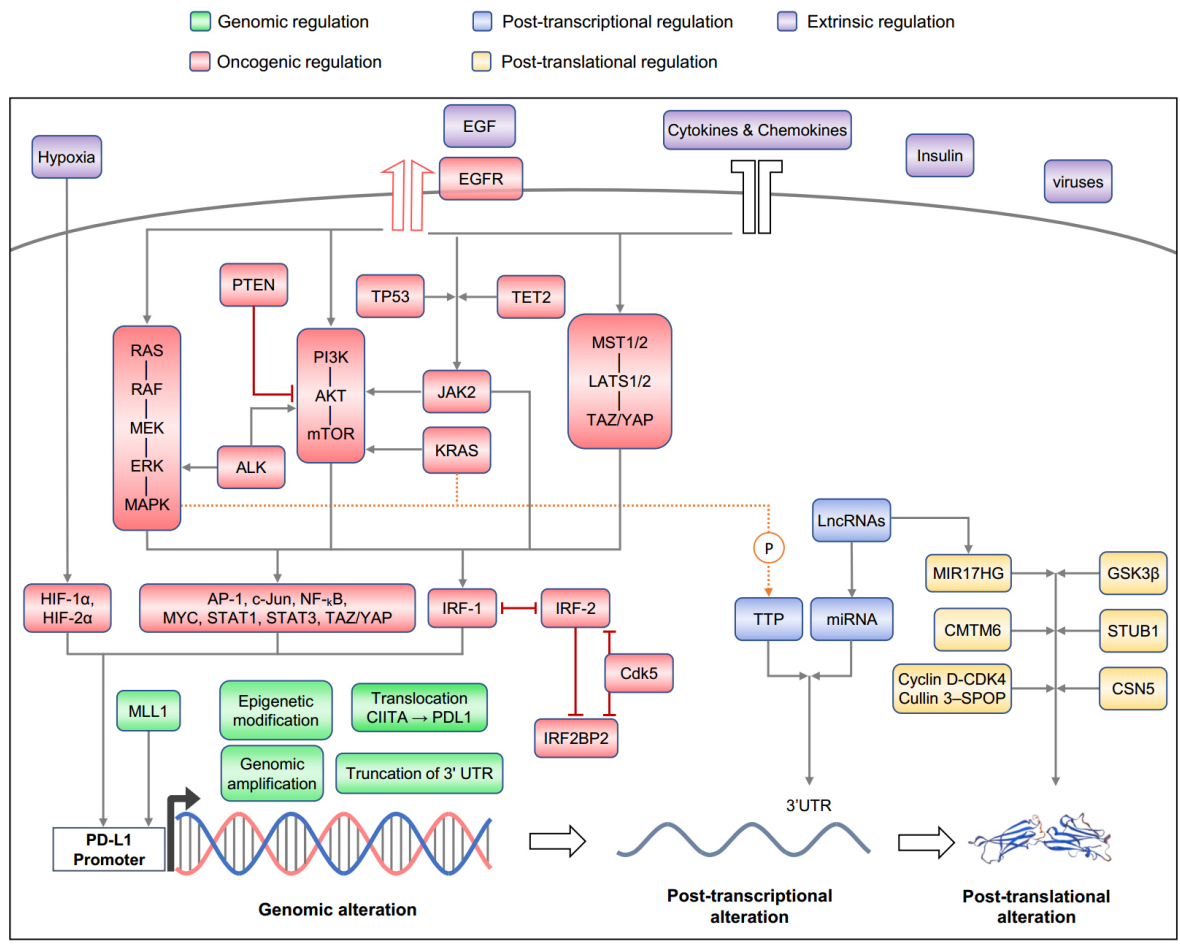

Fig. 1. Overview of the regulatory mechanisms of PD-L1 expression. Solid boxes with different colors represent key molecules/pathways that are differentially involved in the regulation of PD-L1 expression. be a combinatorial approach to the current PD-1/PD-L1 blockade therapy.

\section{INTRINSIC ACTIVITY OF PD-L1}

PD-L1 was not believed to have a canonical signaling motif, but recently PD-L1 was proposed to contain unconventional motifs that are responsible for the resistance to IFN- $\gamma$-mediated cytotoxicity (45), an observation that will open up the path to a comprehensive understanding of protumoral activity of PD-L1. We summarize the current knowledge of the role of cancerintrinsic PD-L1 in protumoral activities.

\section{Epithelial-to-mesenchymal transition (EMT)}

EMT is a cellular process where epithelial cells are transformed into mesenchymal-like cells with migratory and invasive activity. This involves the changes in the expression of several key EMT markers during the process, with downregulation of E-cadherin and upregulation of $\mathrm{N}$-cadherin, fibronectin, vimentin, snail, slug, twist, and zeb1 (46).

Cao et al. first reported that methylcholanthrene (MCA)-induced squamous-cell carcinoma derived from PD-L1 transgenic mice downregulated the E-cadherin transcript, but upregulated EMT-related transcription factors, including slug and twist, more than in mice without PD-L1 expression, indicating that PD-L1 can regulate EMT process (47). Subsequent studies have revealed that PD-L1 can control the signaling pathways that lead to EMT. PD-L1 promotes migration and stemness in renal-cell carcinoma by regulating SREBP-1c expression, a transcription factor that increases cancer-cell migration (48). PD-L1 also activates EMT process by interacting with $\mathrm{H}$-Ras, which leads to activation of the Ras/MEK/ERK pathway, not the PI3K/AKT pathway, in glioblastoma multiforme (49). Depending on the context, PD-L1 can also promote EMT by means of the PI3KJAKT pathway. PD-L1 knockdown lessened the expression of $\mathrm{N}$-cadherin and vimentin with reduced phosphorylated AKT in hypopharyngeal squamous-cell carcinoma (50), and overexpressed PD-L1 in turn augmented migration and invasion of nasopharyngeal-cell carcinoma by activating the PI3K/AKT pathway (51). In addition, PD-L1 was reported to promote migration and invasion by upregulating Smad3 phosphorylation that leads to the activation of the TGF- $\beta / S m a d$ pathway in EGFR-mutant NSCLC with parallel resistance to gefitinib, an EGFR tyrosine kinase inhibitor (52). PD-L1 expression also facilitates the proliferation and migration of multiple cancers by phosphorylation of mTORC1 substrate $\mathrm{p} 70 \mathrm{~S} 6 \mathrm{~K}^{\mathrm{T} 389}$, thereby inducing mTORC1 activity (53). The $\mathrm{PI} 3 \mathrm{~K} / \mathrm{AKT} / \beta$-catenin/WIP pathway is important in PD-L1-mediated EMT induction, where PD-L1 upregulates $\beta$-catenin and subsequently promotes $\beta$-catenin binding to WIP promoter, leading to the expression of the WIP protein that affects the actin polymerization (6).

\section{Cancer metabolism}

Tumor metabolism is a critical element that can help tumor 
cells to survive in a hostile environment, including low oxygen tension and nutrient competition with tumor-infiltrating lymphocytes. Cancers circumvent these settings by evolving an adaptive metabolic process where they increase aerobic glycolysis, alter mitochondrial metabolism, and increase amino-acid uptake (54).

A growing number of studies has suggested that PD-L1 is directly or indirectly involved in cancer-cell metabolism. In a study using a mouse sarcoma model, PD-L1 knockdown reduced the extracellular acidification rate (ECAR), inhibited AKT and mTOR activity, and decreased the expression of glycolysis enzymes, reflecting PD-L1's role in cancer glucose use by means of the ATK/mTOR pathway (55). In another study, PD-L1 directly bound to integrin $\beta 4$ (ITGB4), activated the AKT/GSK3 $\beta$ signaling pathway, and subsequently induced transcriptional repressor SNAI1, which further inhibited SIRT3 promoter activity, thus increasing EMT and tumor glucose uptake (56). Recently, the idea that cancer-intrinsic PD-L1 facilitates cancer glucose metabolism in NSCLC was further backed up by the observation that knockdown of PD-L1 decreased the expression of hexokinase-2 (HK2), an enzyme phosphorylating glucose, to produce glucos-6-phosphate and downregulated glycolysis by inhibiting the PI3K/AKT and ERK pathways (57).

In addition to cancer glucose metabolism, PD-L1 was reported to increase cancer lipid uptake by upregulating fatty-acid binding protein (Fabp) 4 and 5 expression in gastric adenocarcinoma. In TME, highly proliferating cancer cells that compete with tissue-resident memory $\mathrm{T}$ (Trm) cells for glucose and lipid exploit PD-L1 to promote glucose and lipid metabolism so that they acquire a selective advantage over Trm cells, leading to the evasion of anti-tumor immunity (58).

\section{Cancer stemness}

Cancer stem cells (CSCs) are a subpopulation of cancer cells that possess characteristics of normal stem cells, capable of indefinite self-renewal, differentiation into multiple cell types, and resistance to therapies, including radiation and chemotherapy (59).

In a previous study, a subpopulation of cholangiocarcinoma cell lines with low PD-L1 expression was depicted as CSCs on the basis of observation that they showed higher ALDH and lower reactive oxygen species activities, a characteristics of CSC, and highly expressed mRNA of stemness-associated genes, including Nanog, Sox2, and Oct-3/4, compared to cells with high PD-L1 levels, indicating the potential role of PD-L1 in suppression of tumorigenesis (60). However, many recent studies have identified cancer-intrinsic PD-L1 as being associated with sustaining cancer stemness. A growing body of evidence linked PD-L1 expression to cancer stemness by comparing the PD-L1 expression in CSCs and non-CSCs that are isolated from bladder, breast, and colon cancers $(61,62)$. RNA interference-based studies have revealed that PD-L1 directly regulates the expression of the embryonic stem-cell transcription factors, including Oct-4A, Nanog, and the stemness factor BMI1 $(63,64)$. Mecha- nistically, PD-L1 knockdown disrupts phosphorylation of AKT and $\mathrm{p}$-S6 that leads to inhibition of the PI3K/AKT/mTOR pathway and eventually culminates in abolished Oct-4A phosphorylation, indicating that PD-L1 regulates Oct-4A function via the PI3K/AKT/mTOR pathway (63). In CD133 ${ }^{+} \mathrm{CD} 44^{+}$colorectal CSCs, overexpression or knockdown of PD-L1 has revealed that PD-L1 increases and directly binds to a high-mobility group protein called HMGA1, and promotes expression of stemness-associated genes, including Oct-4, ABCG2, ALDH1, and Bmi-1, by means of the MEK/ERK and AKT pathways (65).

We reported that PD-L1-positive myeloma cells were more likely to be resistant to apoptosis induced by melphalan and bortezomib when they were treated with soluble PD-1 fusion protein. This PD-L1 engagement induced activation of the PI3K-AKT pathway, leading to the resistance of myeloma cells to apoptosis against the drugs (66). In support of these findings, PD-L1-expressing breast and prostate cancer cells manifest resistance to doxorubicin and docetaxel upon PD-L1 engagement with a soluble PD-1 fusion protein or membrane PD-1 expressed on Jurkat $T$ cells by means of the activated ERK and mTOR pathways (67). In another study, ligation of cancer PD-L1 with PD-1 fusion protein increased multidrug resistance 1/P-glycoprotein (MDR1/P-gp) by phosphorylating AKT and ERK that leads to the activation of the PI3K-AKT and MAPK-ERK pathways, rendering the cells resistant to doxorubicin (68). It should be noted that cancer-intrinsic PD-L1 can make cancer cells more pro-apoptotic in a context-dependent way. For example, deletion of PD-L1 made CRC cells resistant to apoptosis against cytotoxic drugs by suppressing the $\mathrm{BH} 3$-only proteins $\mathrm{BIK}$ and BIM, an activator for pro-apoptotic proteins (69).

In a recent study, PD-L1 was described as an RNA-binding protein protecting mRNAs from DNA-damage-related genes, including NBS1 and BRCA1, from degradation, thereby leading to the resistance to radiation or chemotherapy (70). Current findings indicate that PD-L1 may function as an oncoprotein that can regulate cancer survival, proliferation, and resistance.

\section{Autophagy}

Autophagy is a cellular process that primarily responds to long-term nutrient deprivation, preserving the healthy cells and tissues by removing the damaged cells/organelles, a process essential for maintenance of cellular homeostasis. Recent studies indicate that autophagy plays a dual role in tumor progression as a tumor suppressor and a tumor increaser, depending on the context (71).

Autophagy is known to regulate PD-L1 expression in certain cancer types, whereas the autophagic process is also believed to be regulated by cancer-intrinsic PD-L1. The findings that increased PD-L1 expression inhibits autophagy in multiple cell lines have been demonstrated in recent studies using mouse melanoma and ovarian cancer (OC) cell lines (72). In a study using a glioblastoma multiforme (GBM) model, upregulated PD-L1 in the frontier GMB area with relatively less severe starvation binds to the non-PH domain of AKT in the cytosol 


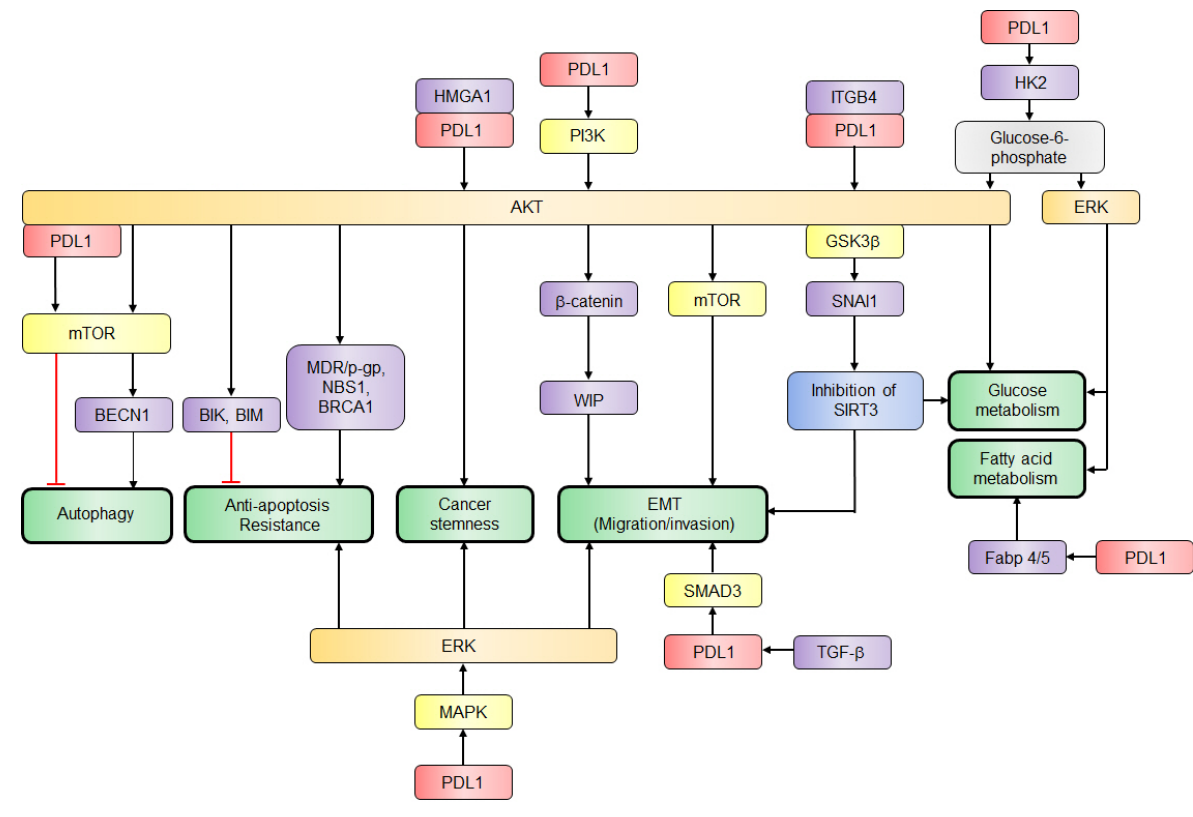

Fig. 2. Overview of protumoral activity of cancer-intrinsic PD-L1. Green boxes with bold lines represent intrinsic activities of PD-L1 and the rest of solid boxes with different colors indicate key molecules/pathways that can lead to each cancer intrinsic activity of PD-L1. and facilitates its translocation to the plasma membrane to fully activate AKT, leading to the suppression of autophagy. In this model, PD-L1 increases F-actin formation and colocalization of AKT and F-actin underneath plasma membrane and facilitates GBM invasion under starvation stress (73). However, in another study, PD-L1 was described as increasing the autophagy by upregulating BECN1, a regulator of autophagy (74). The discrepancy between these results requires a further mechanistic study of the role of cancer-intrinsic PD-L1 in the regulation of autophagy.

\section{The intracellular signalosome of cancer-intrinsic PD-L1}

Despite the lack of canonical signaling motifs on the PD-L1 sequence, PD-L1 can form intracellular signalosomes that are associated with survival, stress responses, and resistance to pro-apoptotic stimuli. Recently, Gato-Canas and colleagues identified two signal transduction functional motifs on the PD-L1 intracellular domain that are responsible for cancer-cell growth and resistance to interferons (45). From the alignment analysis of 10 mammalian PD-L1 sequences, they identified three phylogenetically conserved sequence motifs, termed "RMLDVEKC", "DTSSK", and "QFEET". Among them, "RMLDVEKC" is critical for cancer resistance to cytotoxicity of type I and II IFNs, whereas the "DTSSK" motif behaves as a negative regulator for "RMLDVEKC", leading to sensitivity toward IFNs. Analysis of a public cancer database, including TCGA, COSMIC, ICGC, and Intogen, has revealed that somatic mutations in the "DTSSK" motif was found in skin and lung cancers (45). Furthermore, mutations in a "DTSSK" mouse motif protected mouse cancer cells from IFN's cytotoxicity, implying the importance of "DTSSK" mutations in clinical outcome of cancer immuno- therapy where IFNs act as key effector molecules.

Comprehensive interactome analysis of PD-L1 using high-throughput affinity-purification mass spectrometry identified a signalosome group that consists of the mTOR pathway, regulators of the DNA damage response (DDR) pathway, such as ATM, ATR, PDS5B, and TTL1, and kinases involved in survival and anchorage-independent cell growth $(75,76)$. STRING analysis further identified the possible crosstalk between the PD-L1 interactome and mediator of the IFN signaling pathway. The observation that cancer-intrinsic PD-L1 regulates DDR, tumorigenesis, and resistance to therapy may be explained by crosstalk between PD-L1 and signalosomes with some kinases as regulatory nodes.

Collectively, a growing body of evidence suggests that PD-L1 is a fascinating target for cancer therapy, because it plays a dual role in anti-tumor immunity by shutting down the T cell immunity as well as by turning on the protumoral program. This perspective may explain more clearly the contribution of cancer-intrinsic PD-L1 to the worse prognosis of cancer patients with higher expression of PD-L1 (Fig. 2). Particularly, the molecules and signaling pathways that are engaged in the protumoral activity of cancer-intrinsic PD-L1 might provide the clues for the non-responsiveness to PD-1/PD-L1 inhibitor therapy. In addition, comprehensive understanding of the biology of cancerintrinsic PD-L1 would set the stage for the development of a new therapeutic approach by targeting the molecular network formed by cancer-intrinsic PD-L1.

\section{ACKNOWLEDGEMENTS}

This work was supported by the 2019 Inje University Research 
Grant.

\section{CONFLICTS OF INTEREST}

The authors have no conflicting interests.

\section{REFERENCES}

1. Chen L and Flies DB (2013) Molecular mechanisms of T cell co-stimulation and co-inhibition. Nat Rev Immunol 13, 227-242

2. Cai J, Wang D, Zhang G and Guo X (2019) The role of PD-1/PD-L1 axis in treg development and function: implications for cancer immunotherapy. Onco Targets Ther 12, 8437-8445

3. Xin Yu J, Hodge JP, Oliva C, Neftelinov ST, HubbardLucey VM and Tang J (2020) Trends in clinical development for PD-1/PD-L1 inhibitors. Nat Rev Drug Discov 19, 163-164

4. Sun L, Zhang L, Yu J et al (2020) Clinical efficacy and safety of anti-PD-1/PD-L1 inhibitors for the treatment of advanced or metastatic cancer: a systematic review and meta-analysis. Sci Rep 10, 2083

5. Azuma T, Yao S, Zhu G, Flies AS, Flies SJ and Chen L (2008) B7-H1 is a ubiquitous antiapoptotic receptor on cancer cells. Blood 111, 3635-3643

6. Yu W, Hua Y, Qiu H et al (2020) PD-L1 promotes tumor growth and progression by activating WIP and beta-catenin signaling pathways and predicts poor prognosis in lung cancer. Cell Death Dis 11, 506

7. Lee S-J, Jang B-C, Lee S-W et al (2006) Interferon regulatory factor- 1 is prerequisite to the constitutive expression and IFN- $\gamma$-induced upregulation of $\mathrm{B} 7-\mathrm{H} 1$ (CD274). FEBS Lett 580, 755-762

8. Cancer Genome Atlas Research Network (2017) Integrated genomic and molecular characterization of cervical cancer. Nature 543, 378-384

9. Steidl C, Shah SP, Woolcock BW et al (2011) MHC class II transactivator CIITA is a recurrent gene fusion partner in lymphoid cancers. Nature 471, 377-381

10. Kataoka K, Shiraishi Y, Takeda Y et al (2016) Aberrant PD-L1 expression through $3^{\prime}$-UTR disruption in multiple cancers. Nature 534, 402-406

11. Wu Y, Zhao T, Jia Z et al (2019) Polymorphism of the programmed death-ligand 1 gene is associated with its protein expression and prognosis in gastric cancer. J Gastroenterol Hepatol 34, 1201-1207

12. Xiao G, Jin L-L, Liu C-Q et al (2019) EZH2 negatively regulates PD-L1 expression in hepatocellular carcinoma. J Immunother Cancer 7, 1-15

13. Nair VS, El Salhat H, Taha RZ, John A, Ali BR and Elkord E (2018) DNA methylation and repressive H3K9 and $\mathrm{H} 3 \mathrm{~K} 27$ trimethylation in the promoter regions of PD-1, CTLA-4, TIM-3, LAG-3, TIGIT, and PD-L1 genes in human primary breast cancer. Clin Epigenetics 10, 78

14. Lu C, Paschall AV, Shi H et al (2017) The MLL1-H3K4me3 axis-mediated PD-L1 expression and pancreatic cancer immune evasion. J Natl Cancer Inst 109, djw283

15. Casey SC, Tong L, Li Y et al (2016) MYC regulates the antitumor immune response through CD47 and PD-L1. Science 352, 227-231

16. Song TL, Nairismägi M-L, Laurensia $Y$ et al (2018) Oncogenic activation of the STAT3 pathway drives PD-L1 expression in natural killer/T-cell lymphoma. Blood 132, 11461158

17. Ruf M, Moch H and Schraml P (2016) PD-L1 expression is regulated by hypoxia inducible factor in clear cell renal cell carcinoma. Int J Cancer 139, 396-403

18. Noman MZ, Desantis G, Janji B et al (2014) PD-L1 is a novel direct target of HIF- $1 \alpha$, and its blockade under hypoxia enhanced MDSC-mediated T cell activation. J Exp Med 211, 781-790

19. Green MR, Rodig S, Juszczynski P et al (2012) Constitutive AP-1 activity and EBV infection induce PD-L1 in Hodgkin lymphomas and posttransplant lymphoproliferative disorders: implications for targeted therapy. Clin Cancer Res 18, 1611-1618

20. Liu J, Hamrouni A, Wolowiec D et al (2007) Plasma cells from multiple myeloma patients express B7-H1 (PD-L1) and increase expression after stimulation with IFN- $\gamma$ and TLR ligands via a MyD88-, TRAF6-, and MEK-dependent pathway. Blood 110, 296-304

21. Stutvoet TS, Kol A, de Vries EG et al (2019) MAPK pathway activity plays a key role in PD-L1 expression of lung adenocarcinoma cells. J Pathol 249, 52-64

22. Coelho MA, de Carné Trécesson S, Rana S et al (2017) Oncogenic RAS signaling promotes tumor immunoresistance by stabilizing PD-L1 mRNA. Immunity 47, 1083-1099

23. Hong S, Chen N, Fang W et al (2016) Upregulation of PD-L1 by EML4-ALK fusion protein mediates the immune escape in ALK positive NSCLC: implication for optional anti-PD-1/PD-L1 immune therapy for ALK-TKIs sensitive and resistant NSCLC patients. Oncoimmunology 5, e1094598

24. van Rensburg HJJ, Azad T, Ling M et al (2018) The Hippo pathway component TAZ promotes immune evasion in human cancer through PD-L1. Cancer Res 78, 1457-1470

25. Chen J, Zhang XD and Proud C (2015) Dissecting the signaling pathways that mediate cancer in PTEN and LKB1 double-knockout mice. Sci Signal 8, pe1

26. Thiem A, Hesbacher S, Kneitz $\mathrm{H}$ et al (2019) IFN-gammainduced PD-L1 expression in melanoma depends on p53 expression. J Exp Clin Cancer Res 38, 1-15

27. Cortez MA, Ivan C, Valdecanas D et al (2016) PDL1 regulation by p53 via miR-34. J Natl Cancer Inst 108, djv303

28. Xu Y-p, Lv L, Liu Y et al (2019) Tumor suppressor TET2 promotes cancer immunity and immunotherapy efficacy. J Clin Invest 129, 4316-4331

29. Wu A, Wu Q, Deng Y et al (2019) Loss of VGLL 4 suppresses tumor PD-L1 expression and immune evasion. EMBO J 38, e99506

30. Yan Y, Zheng L, Du Q, Yan B and Geller DA (2020) Interferon regulatory factor 1 (IRF-1) and IRF-2 regulate PD-L1 expression in hepatocellular carcinoma (HCC) cells. Cancer Immunol Immunother 69, 891-1903

31. Dorand RD, Nthale J, Myers JT et al (2016) Cdk5 disruption attenuates tumor PD-L1 expression and promotes antitumor immunity. Science 353, 399-403

32. Chen L, Liu S and Tao Y (2020) Regulating tumor suppressor genes: post-translational modifications. Signal Transduct 
Target Ther 5, 90

33. Wei S, Wang K, Huang X, Zhao Z and Zhao Z (2019) LnCRNA MALAT1 contributes to non-small cell lung cancer progression via modulating miR-200a-3p/programmed deathligand 1 axis. Int J Immunopathol Pharmacol 33, 2058738 419859699

34. Zhao L, Liu Y, Zhang J, Liu Y and Qi Q (2019) LncRNA SNHG14/miR-5590-3p/ZEB1 positive feedback loop promoted diffuse large $B$ cell lymphoma progression and immune evasion through regulating PD-1/PD-L1 checkpoint. Cell Death Dis 10, 1-15

35. Mineo M, Lyons SM, Zdioruk M et al (2020) Tumor interferon signaling is regulated by a IncRNA INCR1 transcribed from the PD-L1 Locus. Mol Cell 78, 1209-1223

36. Li CW, Lim SO, Xia W et al (2016) Glycosylation and stabilization of programmed death ligand-1 suppresses T-cell activity. Nat Commun 7, 12632

37. Chan LC, Li CW, Xia W et al (2019) IL-6/JAK1 pathway drives PD-L1 Y112 phosphorylation to promote cancer immune evasion. J Clin Invest 129, 3324-3338

38. Apriamashvili G, Vredevoogd DW, Krijgsman $\mathrm{O}$ et al (2020) Loss of ubiquitin ligase STUB1 amplifies IFN $\gamma-\mathrm{R} 1 /$ JAK1 signaling and sensitizes tumors to IFN $\gamma$. bioRxiv, https:// doi.org/10.1101/2020.07.07.191650

39. Mezzadra R, Sun C, Jae LT et al (2017) Identification of CMTM6 and CMTM4 as PD-L1 protein regulators. Nature $549,106-110$

40. Burr ML, Sparbier CE, Chan Y-C et al (2017) CMTM6 maintains the expression of PD-L1 and regulates anti-tumour immunity. Nature 549, 101-105

41. Lim SO, Li CW, Xia W et al (2016) Deubiquitination and stabilization of PD-L1 by CSN5. Cancer Cell 30, 925-939

42. Zhang J, Bu X, Wang $\mathrm{H}$ et al (2019) Author correction: cyclin D-CDK4 kinase destabilizes PD-L1 via cullin 3-SPOP to control cancer immune surveillance. Nature 571, E10

43. Xu J, Meng Q, Li X et al (2019) Long noncoding RNA MIR17HG promotes colorectal cancer progression via miR17-5p. Cancer Res 79, 4882-4895

44. Zhang M, Wang N, Song P et al (2020) LncRNA GATA3AS1 facilitates tumour progression and immune escape in triple-negative breast cancer through destabilization of GATA3 but stabilization of PD-L1. Cell Prolif 53, e12855

45. Gato-Canas M, Zuazo M, Arasanz H et al (2017) PDL1 signals through conserved sequence motifs to overcome interferon-mediated cytotoxicity. Cell Rep 20, 1818-1829

46. Dongre A and Weinberg RA (2019) New insights into the mechanisms of epithelial-mesenchymal transition and implications for cancer. Nat Rev Mol Cell Biol 20, 69-84

47. Cao Y, Zhang L, Kamimura Y et al (2011) B7-H1 overexpression regulates epithelial-mesenchymal transition and accelerates carcinogenesis in skin. Cancer Res 71, 1235-1243

48. Wang Y, Wang H, Zhao Q, Xia Y, Hu X and Guo J (2015) PD-L1 induces epithelial-to-mesenchymal transition via activating SREBP-1c in renal cell carcinoma. Med Oncol 32,212

49. Qiu XY, Hu DX, Chen WQ et al (2018) PD-L1 confers glioblastoma multiforme malignancy via Ras binding and Ras/Erk/EMT activation. Biochim Biophys Acta Mol Basis Dis $1864,1754-1769$

50. Cui $P$, Jing $P$, Liu $X$ and $X u$ W (2020) Prognostic signifi- cance of PD-L1 expression and its tumor-intrinsic functions in hypopharyngeal squamous cell carcinoma. Cancer Manag Res 12, 5893-5902

51. Fei Z, Deng Z, Zhou L, Li K, Xia X and Xie R (2019) PD-L1 induces epithelial-mesenchymal transition in nasopharyngeal carcinoma cells through activation of the PI3K/AKT Pathway. Oncol Res 27, 801-807

52. Zhang $Y$, Zeng $Y$, Liu $T$ et al (2019) The canonical TGF-beta/Smad signalling pathway is involved in PD-L1-induced primary resistance to EGFR-TKIs in EGFR-mutant non-small-cell lung cancer. Respir Res 20, 164

53. Clark CA, Gupta HB, Sareddy G et al (2016) Tumor-intrinsic PD-L1 signals regulate cell growth, pathogenesis, and autophagy in ovarian cancer and melanoma. Cancer Res 76, 6964-6974

54. Martinez-Outschoorn UE, Peiris-Pagés M, Pestell RG, Sotgia F and Lisanti MP (2017) Cancer metabolism: a therapeutic perspective. Nat Rev Clin Oncol 14, 11-31

55. Chang CH, Qiu J, O'Sullivan D et al (2015) Metabolic competition in the tumor microenvironment is a driver of cancer progression. Cell 162, 1229-1241

56. Wang S, Li J, Xie J et al (2018) Programmed death ligand 1 promotes lymph node metastasis and glucose metabolism in cervical cancer by activating integrin beta4/SNAI1/SIRT3 signaling pathway. Oncogene 37, 4164-4180

57. Kim S, Jang JY, Koh J et al (2019) Programmed cell death ligand-1-mediated enhancement of hexokinase 2 expression is inversely related to T-cell effector gene expression in non-small-cell lung cancer. J Exp Clin Cancer Res 38, 462

58. Lin R, Zhang $\mathrm{H}$, Yuan $\mathrm{Y}$ et al (2020) Fatty acid oxidation controls CD8 $(+)$ tissue-resident memory T-cell survival in gastric adenocarcinoma. Cancer Immunol Res 8, 479-492

59. Najafi M, Farhood B and Mortezaee K (2019) Cancer stem cells (CSCs) in cancer progression and therapy. J Cell Physiol 234, 8381-8395

60. Tamai K, Nakamura M, Mizuma M et al (2014) Suppressive expression of CD274 increases tumorigenesis and cancer stem cell phenotypes in cholangiocarcinoma. Cancer Sci 105, 667-674

61. Jinesh GG, Manyam GC, Mmeje CO, Baggerly KA and Kamat AM (2017) Surface PD-L1, E-cadherin, CD24, and VEGFR2 as markers of epithelial cancer stem cells associated with rapid tumorigenesis. Sci Rep 7, 9602

62. Wu Y, Chen M, Wu P, Chen C, Xu ZP and Gu W (2017) Increased PD-L1 expression in breast and colon cancer stem cells. Clin Exp Pharmacol Physiol 44, 602-604

63. Almozyan S, Colak D, Mansour F et al (2017) PD-L1 promotes OCT4 and Nanog expression in breast cancer stem cells by sustaining PI3K/AKT pathway activation. Int J Cancer 141, 1402-1412

64. Zhang X, Li F, Zheng Y et al (2019) Propofol reduced mammosphere formation of breast cancer stem cells via PD-L1/Nanog in vitro. Oxid Med Cell Longev 2019, 9078209

65. Wei F, Zhang T, Deng SC et al (2019) PD-L1 promotes colorectal cancer stem cell expansion by activating HMGA1dependent signaling pathways. Cancer Lett 450, 1-13

66. Ishibashi M, Tamura H, Sunakawa M et al (2016) Myeloma drug resistance induced by binding of myeloma $\mathrm{B} 7-\mathrm{H} 1$ (PD-L1) to PD-1. Cancer Immunol Res 4, 779-788 
67. Black M, Barsoum IB, Truesdell P et al (2016) Activation of the PD-1/PD-L1 immune checkpoint confers tumor cell chemoresistance associated with increased metastasis. Oncotarget 7, 10557-10567

68. Liu S, Chen S, Yuan W et al (2017) PD-1/PD-L1 interaction up-regulates MDR1/P-gp expression in breast cancer cells via PI3K/AKT and MAPK/ERK pathways. Oncotarget 8, 99901-99912

69. Feng D, Qin B, Pal K et al (2019) BRAF(V600E)-induced, tumor intrinsic PD-L1 can regulate chemotherapy-induced apoptosis in human colon cancer cells and in tumor xenografts. Oncogene 38, 6752-6766

70. Tu X, Qin B, Zhang Y et al (2019) PD-L1 (B7-H1) Competes with the RNA exosome to regulate the DNA damage response and can be targeted to sensitize to radiation or chemotherapy. Mol Cell 74, 1215-1226 e1214

71. Brech A, Ahlquist T, Lothe RA and Stenmark H (2009)
Autophagy in tumour suppression and promotion. Mol Oncol 3, 366-375

72. Clark CA, Gupta HB and Curiel TJ (2017) Tumor cell-intrinsic CD274/PD-L1: a novel metabolic balancing act with clinical potential. Autophagy 13, 987-988

73. Chen RQ, Xu XH, Liu F et al (2019) The binding of PD-L1 and Akt facilitates glioma cell invasion upon starvation via Akt/Autophagy/F-Actin signaling. Front Oncol 9, 1347

74. Gao H, Zhang J and Ren X (2019) PD-L1 regulates tumorigenesis and autophagy of ovarian cancer by activating mTORC signaling. Biosci Rep 39, BSR20191041

75. Huttlin EL, Ting L, Bruckner RJ et al (2015) The BioPlex network: a systematic exploration of the human interactome. Cell 162, 425-440

76. Escors D, Gato-Canas M, Zuazo M et al (2018) The intracellular signalosome of PD-L1 in cancer cells. Signal Transduct Target Ther 3, 26 